\title{
Multi-axial non-contact in situ micromanipulation by steady streaming around two oscillating cylinders on holonomic miniature robots
}

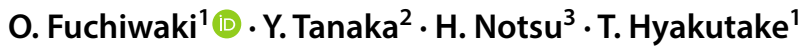

Received: 17 April 2018 / Accepted: 9 July 2018 / Published online: 20 July 2018

(c) Springer-Verlag GmbH Germany, part of Springer Nature 2018

\begin{abstract}
In the field of micromanipulation, an in situ three-axial rotation of a microscale object remains difficult to realize, with rotational resolution and repeatability remaining low. In this paper, we describe the fundamental principle, properties, and experimental results of multi-axial non-contact in situ micromanipulation of an egg cell driven by steady streaming generated around an oscillating cylinder. A continuously oscillating cylinder generates the steady streaming that draws an egg cell toward the cylinder. If it is trapped by an eddy near the tip of the cylinder, it continuously rotates around the vertical axis at a fixed point. If it is trapped by a swirl flow generated around the side of the cylinder, it rotates around the horizontal axis. We define Reynolds number, $R_{\mathrm{e}}$, as $a r_{\mathrm{c}} \omega / \nu$, where $a$ is half of the oscillation's amplitude, $r_{\mathrm{c}}$ is the cylinder's radius, $\omega$ is the oscillation's angular frequency, and $\nu$ is the kinematic viscosity. We demonstrate that the conditions of the vertical and horizontal rotations are determined by two dimensionless numbers: $R_{\mathrm{e}}$ and $a / r_{\mathrm{c}}$. In our experiments, we obtained rotational resolutions of $0.05^{\circ}$ and $0.11^{\circ}$ and maximal angular velocities of $34.8 \%$ and $188 \%$ for the vertical and horizontal rotations, respectively. We also developed unique micromanipulation methods using two oscillating pipettes attached to holonomic miniature robots. We successfully manipulated five degrees of freedom (DoF) of the cell (three posture angles and two translational displacements along the $X$ and $Y$ axes) with the steady streaming. The proposed method enables a multi-axial, non-contact, in situ, and compact micromanipulation independent of the electrical, optical, magnetic, shape, and stiffness properties of the objects; moreover, it can be applied in microfluidics, biomedical, and heterogeneous microassembly applications.
\end{abstract}

Keywords In situ multi-axial micromanipulation $\cdot$ Steady streaming $\cdot$ Oscillating cylinder $\cdot$ Holonomic miniature robot

\section{Introduction}

Recently, micromanipulation ranging from $1 \mu \mathrm{m}$ to $1 \mathrm{~mm}$ has generated significant interest (Savia and Koivo 2009; Cecil et al. 2016). Micromanipulations can be categorized as air, vacuum, and liquid, according to the medium in which the microscopic objects are manipulated (Aoyama and Fuchiwaki 2001; Fuchiwaki et al. 2008; Xie and Regnier 2011; Rong et al. 2014).

The micromanipulation in air is important for surface mounting technology (SMT) of electronic chip parts. Owing to a rapid growth in the demand for smaller personal computers, there is an increasing need to reduce the size of electronic components. However, three main problems are associated with micromanipulation in air. One is the "sticky problem;" microscopic objects can be straightforwardly trapped owing to the action of adhesive forces such as the electrostatic force, interfacial force, and/or viscous drag 
force, all of which are generally negligible in manipulations of supra-cm-size objects. The second problem is the challenge of three-axial rotation; it is difficult to control three posture angles of a small object by fixing its central point because manipulators become larger in size and heavy if three rotational stages are added (Hatamura et al. 1995). With mobile precise robots for positioning the micromanipulators, manipulation becomes relatively compact and flexible (Fatikow et al. 2000; Fuchiwaki et al. 2012, 2014), although these mobile robots should be rotated precisely around the central point of the manipulated microscopic object (Fuchiwaki et al. 2015). The third problem is associated with the limitation of the shape of the object; the shape should be cubic because it is convenient to fix two posture angles of a cube around two horizontal axes by putting them on their base plate (Chen et al. 2010; Zhang et al. 2010; Tamadazte et al. 2011; Boudaoud 2012). However, the smaller the objects become, the higher the risk of damaging them or flicking them away by conventional manipulations. These problems can be alleviated using the liquid's capillary force for micromanipulation (Obata et al. 2004; Kumagai and Fuchiwaki 2012; Fuchiwaki and Kumagai 2013; Fan et al. 2015). However, regulating the capillary force remains challenging because small droplets of water (below $1 \mathrm{~mm}$ in size) dry rapidly, and most non-drying liquids introduce contaminations. These problems intensify when micromanipulating complex-shaped and fragile objects.

Micromanipulation in a liquid significantly alleviates these three problems because a liquid flow carries and rotates the immersed microscopic objects and protects them by the liquid layer. Most of the sub-1-mm-size living creatures dwell in water because it is their optimal dwelling medium. Blood vessels are highly effective systems for transportation of nutrients (Hyakutake et al. 2015; Hyakutake and Nagai 2015). When we use grippers for grabbing fragile objects such as biological cells in a liquid, we need to precisely regulate the grasping force; otherwise, the grasped cells will be damaged (Kim et al. 2008; Yabugaki et al. 2013). A significant amount of research has been performed on developing methods for indirect micromanipulation of biological cells in liquids, using optical tweezers (Grier 2003; Chen et al. 2013; Ozkan et al. 2003; Shafiee et al. 2009; Chowdhury et al. 2013), although optical property of the targets affects their manipulating properties. Particle-based micromanipulation by optical or magnetic forces has also been categorized as contact manipulation in liquids (Gijs 2004; Yesin et al. 2006; Floyd et al. 2009; Elbuken et al. 2009). Those techniques can still damage fragile objects.

Acoustic streaming is an interesting phenomenon because it exhibits steady-streaming patterns that change according to the frequency and amplitude of oscillations (Tatsuno 1973, 1981; Wang and Drachman 1982; Sadhai 2012; Nuriev and Zaitseva 2014; Coenen 2016). Recently, we have proposed and studied a method and applications for steady-streaming-based micromanipulation that utilizes an oscillating cylinder (Misaki et al. 2004, 2007; US Patent 2005; Aoyama 2006). The steady streaming is among the preferable methods of non-contact micromanipulation because objects can be manipulated independent of their electrical, optical, magnetic, shape, surface, and stiffness properties. Significant attention has been devoted to this issue (Lieu 2012; Hattori et al. 2015; Leibacher 2015; Amit et al. 2016). Combining acoustic streaming and microscale channels is another potential option for selective transportation of microscale objects (Jeong et al. 2011; Büyükkoçak et al. 2014; Hayakawa et al. 2014; Hayakawa 2015). Several interesting studies have addressed the issue of micromanipulation using acoustic streaming (Friend and Yeo 2011; Walter et al. 2012; Wiklund et al. 2012; Destgeera and Sung 2015).

In the biomedical field, micromanipulation of egg cells is important for realizing transgenic animals and microscale fertilization. No contact micromanipulation using AC-field (Benhal 2014), hydrodynamic flow (Yalikun et al. 2016a, b; Leung et al. 2012), magnetic field-driven rotating particle (Ye et al. 2012) methods are also feasible methods for fragile biomedical cells. In supporting information, Table 1 shows the comparison of those methods with the proposed method for the particle of $\sim 100 \mu \mathrm{m}$ in diameter. AC-field method (Benhal et al. 2014) has $0.57^{\circ}$ of rotational resolution in vertical rotation, although electrical property of the target affects their manipulating property. The hydrodynamic flow method (Yalikun et al. 2016a, b) has advantage for the fast and powerful micromanipulation. The magnetic field-driven rotating particle method (Ye et al. 2012) are usable in closed narrow chamber. However, the in situ three-axial rotation still remains to achieve using these methods, with rotational resolution and repeatability remaining low.

The motivations of this study are to experimentally realize the precise multi-axial in situ rotation of a micro-object by fixing its central point, and to investigate the specifications thorough evaluation of the experimental results from fluid dynamic aspects. The development of a compact manipulating method to demonstrate its feasibility is also a motivation. In this paper, we describe the fundamental principle, properties, and experimental results of the multi-axial micromanipulation of egg cells driven by the steady streaming generated around an oscillating pipette attached immediately above the bottom of a Petri dish. When the cylinder oscillates continuously, the generated acoustic stream draws the microscopic object toward the cylinder. If it is trapped by an eddy in front of the tip of the cylinder, it rotates around the vertical axis. If it is trapped by a swirl flow generated around the side of the cylinder, it rotates around the horizontal axis. For up to one oscillation cycle of the cylinder, the slight eddy or the slight swirl rotates it by a marginal 
rotational angle. In the experiments, we realized non-contact drawing, trapping, and two-axial rotation around the vertical and horizontal axes of the egg cell. In Sect. 2, we explain the condition and the experimental setup. In Sect. 3, we classify the streaming by fluid structures. In Sect. 4, we describe the fundamental performances of the vertical and horizontal rotations and the drawing. In Sect. 5, we describe multi-axial in situ microinjection assisted by oscillating pipettes positioned by holonomic miniature robots (Fuchiwaki 2013) to demonstrate the feasibility. In Sect. 6, we discuss the potential applications in microfluidics and microassembly.

\section{Parameters and experimental setup}

\subsection{Parameters and conditions}

The Reynolds number, $R_{\mathrm{e}}$, is defined as

$R_{\mathrm{e}}=\frac{r_{\mathrm{c}} \cdot a \omega}{v}$,

where $a$ is half of the oscillation's amplitude, $r_{\mathrm{c}}$ is the cylinder's radius, $\omega$ is the oscillation's angular frequency, and $\nu$ is the kinematic viscosity. The cylinder's radius $r_{\mathrm{c}}$ is the characteristic length, and the quantity $a \omega$ is the characteristic velocity. The ratio $a / r_{\mathrm{c}}$ is an important dimensionless parameter that determines the flow patterns as well as $R_{\mathrm{e}}$ (Tatsuno 1973). An example of an acoustic stream around an oscillating straight cylinder is shown in Fig. 1 (Tatsuno 1981). In the caption of Fig. 1, $f$ is the oscillation's frequency. The double arrow indicates the direction of the cylinder's oscillation. The dotted line indicates the bottom of the Petri dish in the present work. Symmetrical circulatory streaming toward

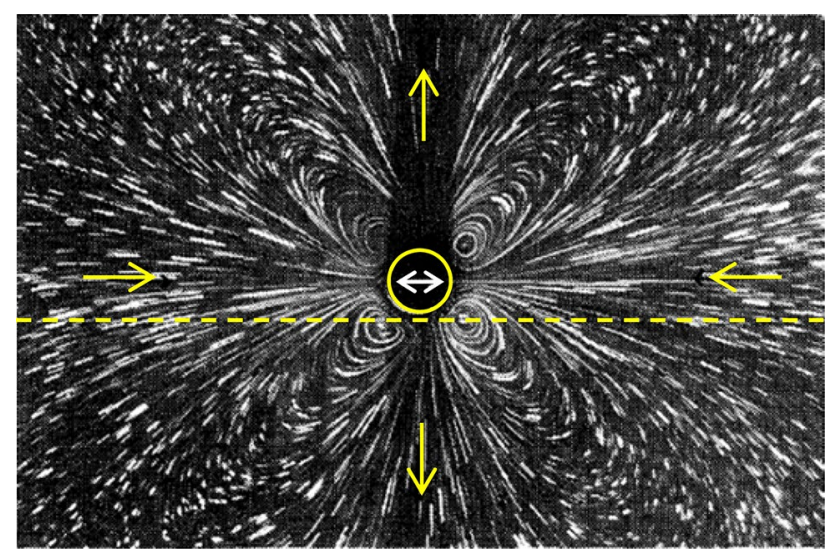

Fig. 1 Image of trajectories of microscopic glass particles with diameters of $\sim 10 \mu \mathrm{m}$, with $\nu=4.15 \times 10^{-5} \mathrm{~m}^{2} / \mathrm{s}, R_{\mathrm{e}}=4.61, a / r_{\mathrm{c}}=0.33$, $f=10 \mathrm{~Hz}, r_{\mathrm{c}}=3020 \mu \mathrm{m}, a=1009 \mu \mathrm{m}$, and exposure time of $10 \mathrm{~s}$. The dotted line indicates the bottom of the container in the present work. Reproduced from ref. Tatsuno (1981) with permission the cylinder along the axis of the oscillation and away from the cylinder perpendicular to the axis is evident. In the present work, we replaced the straight cylinder with a glass pipette that was bent at $40^{\circ}$ and attached immediately above the bottom of the Petri dish filled with pure water. In Sect. 3, we performed experiments to categorize the flow patterns of the acoustic streaming generated by the oscillating bent cylinder, in terms of two dimensionless parameters, $R_{\mathrm{e}}$ and $a / r_{\mathrm{c}}$. In this study, we focused on the micromanipulation of frozen-thawed fertilized egg cells of mice for $r_{\mathrm{e}} / r_{\mathrm{c}}=\sim 1.2$, with $r_{\mathrm{c}}=40 \mu \mathrm{m}$. Here, $r_{\mathrm{e}}$ is defined as the radius of the manipulated egg cell.

\subsection{Experimental setup}

Figure 2a schematically shows the oscillating cylinder driven by a piezoelectric actuator. In the supporting information, Table 2 lists the performance characteristics of the actuator. We denote the oscillating axis as $Y_{\mathrm{P}}$, the horizontal axis perpendicular to $Y_{\mathrm{P}}$ as $X_{\mathrm{P}}$, and the vertical axis as $Z_{\mathrm{P}}$. We used a pipette with an outer diameter of $80 \mu \mathrm{m}$ as the oscillating cylinder. The pipette was bent at $40^{\circ}$ to align it parallel to the bottom. Figure $2 \mathrm{~b}$ shows the experimental setup for observing the steady streaming in the $X_{\mathrm{P}} Y_{\mathrm{P}}$ plane. The oscillating pipette was attached to an $X Y Z$ stage (Eppendorf, 5171). The $X Y Z$ stage was attached to an inverted microscope (Olympus, IX-71). A microscopic image was captured by a high-speed camera (PHOTRON, FAST-CAM NET-C), with the rate of

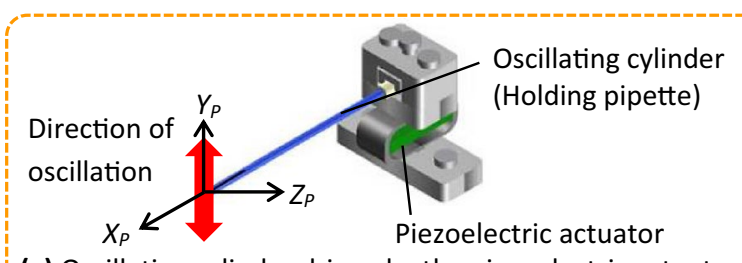

(a) Oscillating cylinder driven by the piezoelectric actuator.

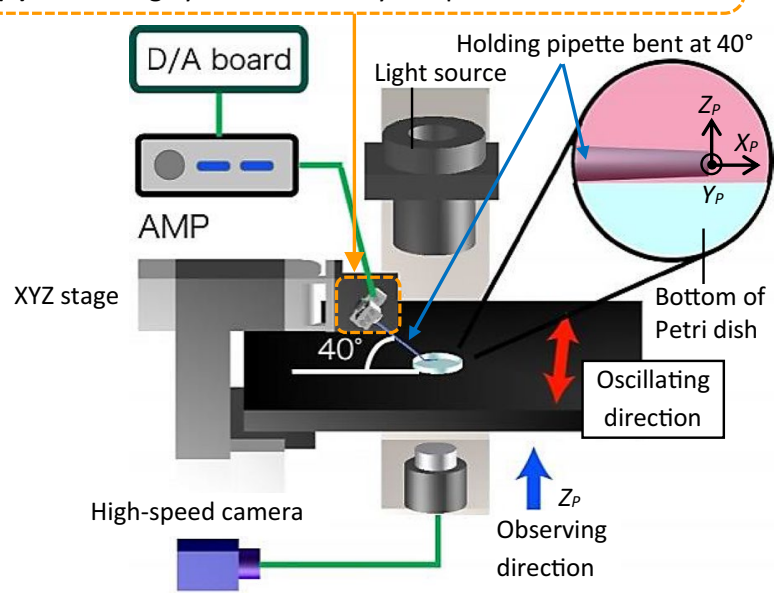

(b) Observing the flow in $X_{P} Y_{P}$ plane using inverted microscopy.

Fig. 2 Experimental setup 
image-capture reaching $250 \mathrm{fps}$, through the bottom of the Petri dish.

The pipette was attached immediately above the bottom, without contact. The oscillation of the pipette was a sinusoidal wave with the half amplitude displacement ranging from 2 to $25 \mu \mathrm{m}$. The displacement was approximately proportional to the applied voltage, although it was affected by the hysteresis-related nonlinearity of the piezoelectric actuator. It was also affected by the viscosity of the liquid and the mechanical resonance frequency; thus, we adjusted the displacement by varying the voltage.

We measured the displacement by the pipette's residual images captured using the fast-speed camera. The microscope's resolution was $0.6 \mu \mathrm{m}$; the measurement errors for the $X$ and $Y$ axes was estimated $\pm 0.3 \mu \mathrm{m}$. The focal depth was $2.9 \mu \mathrm{m}$; the error for the $Z$-axis was estimated $\pm 1.5 \mu \mathrm{m}$. We varied the oscillation frequency from 10 to $250 \mathrm{~Hz}$.

In the experimental work described Sect. 3, a few drops of Indian ink were added as tracer particles into the Petri dish filled with pure water, to investigate the flow patterns. In the work described in Sects. 4 and 5, we put the frozen-thawed fertilized egg cells of mice into the Petri dish filled with a culture solution of M2 to investigate the fundamental manipulating performances.
In Sect. 5.2, we provide additional explanation about the use of the holonomic miniature robots for positioning additional oscillating cylinder.

\section{Flow patterns}

\subsection{Condition of steady streaming}

Figure 3 a shows the diagram of the flow state around the cylinder in the $R_{\mathrm{e}}-a / r_{\mathrm{c}}$ plane for $R_{\mathrm{e}}<1$ and $a / r_{\mathrm{c}}<0.64$. When $R_{\mathrm{e}}>0.12$, we observed the steady streaming. When $R_{\mathrm{e}}<0.08$, the tracer particles oscillated around their initial positions. Although more detailed experimental analysis is necessary to demonstrate the occurrence of the transition with $R_{\mathrm{e}}$, we estimated the transition area to be from 0.08 to 0.12 of $R_{\mathrm{e}}$ for the tracer particles to flow along the streaming.

\subsection{Classification by fluid structure}

Figure $3 b$ shows the typical trajectories of the tracer particles, plotted every $0.1 \mathrm{~s}$ during the window of $0.8 \mathrm{~s}$, with $\left(R_{\mathrm{e}}, a / r_{\mathrm{c}}\right)=(0.49,0.40)$. For $R_{\mathrm{e}}$ in the range $0.12-1.0$, symmetrical steady vertical twin eddies were generated in front

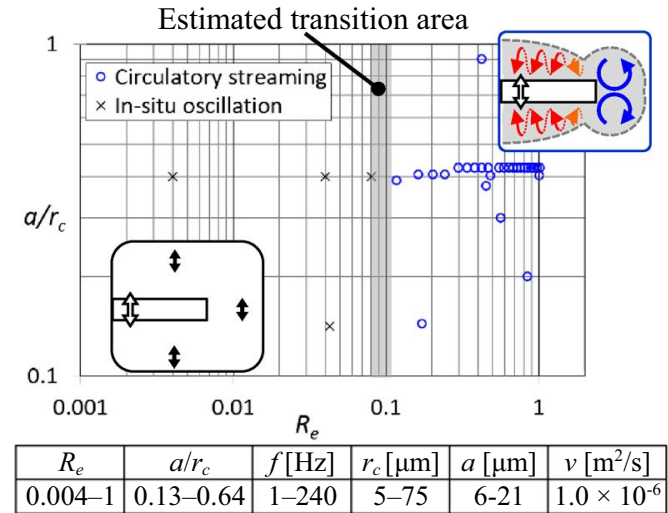

(a) Diagram of the flow state around the cylinder in the $R_{e}-a / r_{c}$ plane.

(c) Classification of the steady streaming and definitions of the symbols and the colors in (b) and (d).

\begin{tabular}{|c|c|c|c|c|}
\hline Symbol & Color & $\begin{array}{l}\text { Region } \\
\text { state }\end{array}$ & \multicolumn{2}{|c|}{$\begin{array}{l}\text { Fluid structure and } \\
\text { direction }\end{array}$} \\
\hline 0 & Blue & \multirow{3}{*}{$\begin{array}{c}\text { Inner } \\
\text { circulatory } \\
\text { streaming }\end{array}$} & \multicolumn{2}{|c|}{ Vertical eddies } \\
\hline 0 & Red & & \multirow{2}{*}{$\begin{array}{l}\text { Horizontal } \\
\text { swirl flows }\end{array}$} & to $-X_{P}$ \\
\hline (2) & Orange & & & to $+X_{P}$ \\
\hline$\Delta$ & Green & \multirow{2}{*}{$\begin{array}{c}\text { Outer } \\
\text { streaming }\end{array}$} & \multirow{2}{*}{$\begin{array}{c}\text { to the } \\
\text { cylinder's }\end{array}$} & side \\
\hline$\frac{\pi}{\lambda}$ & Yellow & & & edge \\
\hline
\end{tabular}

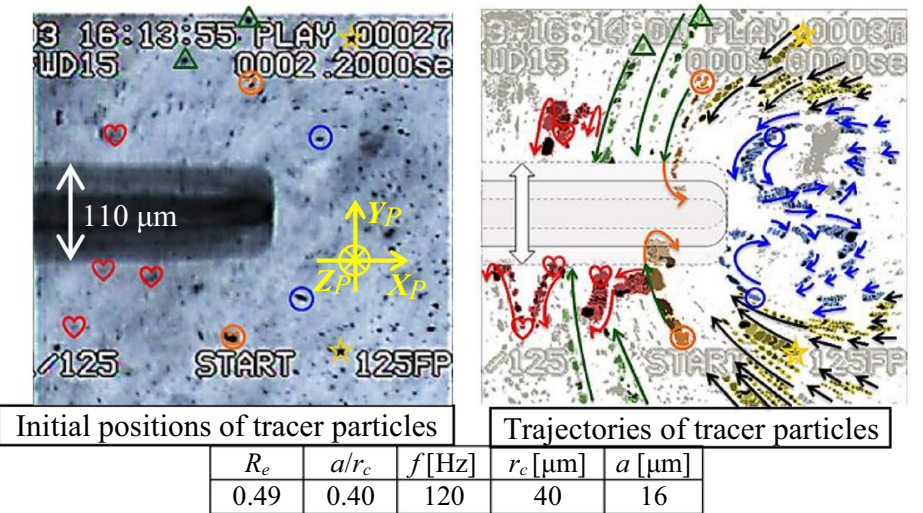

(b) Typical trajectories of tracer particles.
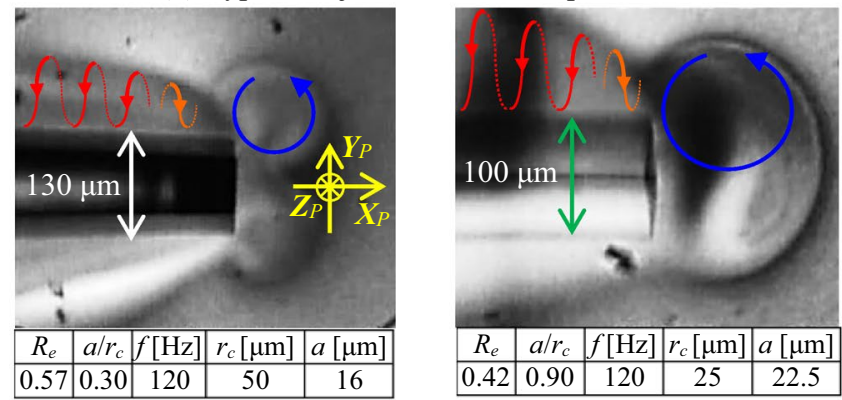

(d) Distribution of tracer particles indicating the layer of the inner circulatory streaming.

Fig. 3 Experimental results of steady-streaming patterns 
of the pipette in the $X_{\mathrm{P}} Y_{\mathrm{P}}$ plane. We hereby call them "vertical eddies".

Symmetrical backward and forward curved flows were also generated around the sides of the pipette in Fig. 3b. We assume that the observed curved flows correspond to the parts of horizontal swirl flows because the flows are approximately half of the axisymmetric circulatory streaming in Fig. 1, if we consider the dotted line to be the bottom of the container. We hereby call them "horizontal swirl flows."

We also observed two types of steady streaming toward the edges and the sides of the cylinder in Fig. 3b. Those two flows correspond to the parts of the streaming toward the cylinder along the axis of the oscillation in Fig. 1.

Figure $3 \mathrm{c}$ shows the classification of the steady streaming by fluid structures and the definitions of the symbols and colors in Fig. 3b, d. We hereby classify "vertical eddies" and "horizontal swirl flows" as "inner circulatory streaming" and the other two streaming as "outer streaming". The inner circulatory streaming and the outer streaming are expected to rotate and draw microscopic objects, respectively.

\subsection{Layer of inner circulatory streaming}

To investigate the shape of the layer of the inner circulatory streaming, we introduced smaller tracer particles of Indian ink into the liquid. Figure $3 \mathrm{~d}$ shows two typical images of their distribution with $\left(R_{\mathrm{e}}, a / r_{\mathrm{c}}\right)=(0.57,0.30)$ and $(0.42$, $0.90)$. The layer was keyhole-shaped, and its thickness varied from 20 to $200 \mu \mathrm{m}$ from the outline of the cylinder.

\section{Fundamental manipulating performances}

\subsection{Vertical rotation}

Figure 4a schematically shows the flow pattern of the vertical rotation. Figure $4 \mathrm{~b}$ shows the motion of the studied egg cell in plots of $a / r_{\mathrm{c}}$ vs. $R_{\mathrm{e}}$. To exhibit the flow patterns without it, the results in Fig. 3a were replotted in Fig. 4b. Three motion patterns are observed in Fig. $4 \mathrm{~b}$. For $R_{\mathrm{e}}$ below 0.07 , it oscillated around its initial position (Mode i). For $R_{\mathrm{e}}$ above 0.07 , it rotated around the vertical axis while fixing the position of the axis (Mode ii). However, for $a / r_{\mathrm{c}}$ above 0.2 and $R_{\mathrm{e}}$ above 0.25 , it was lifted from the bottom and then rolled in the $-X_{\mathrm{P}}$ direction (Mode iii). In the supporting information, Table 3 lists the properties of the vertical rotation without the rolling. Figure $4 \mathrm{c}$ shows snapshots of the two rotation modes.

Figure 4d shows the two typical trajectories of the central point with a vertical rotation, with $\left(R_{\mathrm{e}}, a / r_{\mathrm{c}}\right)=(0.09,0.15)$ and $(0.27,0.11)$. The pair of coordinates $\left(X_{\mathrm{c}}, Y_{\mathrm{c}}\right)$ is the position of the average rotational axis. Figure $4 \mathrm{e}$ shows plots of each of $\omega_{z}, \Delta \theta_{z}$, and $L_{x y} \pm \delta r_{v}$ vs. $R_{\mathrm{e}}$. Here, $\omega_{z}$ is the angular velocity. $\Delta \theta_{z}$ is the rotational resolution during a cycle of the cylinder's oscillation. $L_{x y}$ is the distance between $O$ and $\left(X_{c}, Y_{c}\right) . \delta r_{v}$ is the radial run out and is determined by the maximal distance between $\left(X_{\mathrm{c}}, Y_{\mathrm{c}}\right)$ and the central point. From the left panel of Fig. $4 \mathrm{e}, \omega_{z}$ is approximately proportional to $R_{\mathrm{e}}$. The maximal value of $\omega_{z}$ is $34.8 \%$ for $\left(R_{\mathrm{e}}\right.$, $\left.a / r_{\mathrm{c}}\right)=(0.27,0.11)$. From the middle panel of Fig. $4 \mathrm{e}, \Delta \theta_{z}$ is approximately proportional to $R_{\mathrm{e}}$, and a smaller $a / r_{\mathrm{c}}$ is effective for decreasing $\Delta \theta_{z}$. The minimal value of $\Delta \theta_{z}$ is $0.05^{\circ}$ for $\left(R_{\mathrm{e}}, a / r_{\mathrm{c}}\right)=(0.12,0.05)$. Because of the low $R_{\mathrm{e}}$, it stops immediately after the oscillation stops. This is advantageous for the capability to precisely regulate the rotational angles. In the right panel of Fig. 4e, the error bars are the corresponding $\delta r_{v}$. We observe that both $L_{X Y}$ and $\delta r_{v}$ decrease as $R_{\mathrm{e}}$ increases. We also observe similar characteristics for the form of the two trajectories in Fig. 4d.

For precise and rapid microscopic operations, smaller $a / r_{\mathrm{c}}$ and higher $R_{\mathrm{e}}$ are feasible, owing to the smaller $\Delta \theta_{z}$ and $\delta r_{v}$ and higher $\omega_{z}$. At these values of $\left(R_{\mathrm{e}}, a / r_{\mathrm{c}}\right),(0.27,0.11)$ and $(0.12,0.05)$ should be selected for coarse and precise vertical rotations, respectively.

\subsection{Horizontal rotation}

Figure 5a schematically shows the flow pattern for the horizontal rotation. Figure $5 \mathrm{~b}$ shows the motion patterns for both the vertical and horizontal rotations in the plots of $a / r_{\mathrm{c}} \mathrm{vs}$. $R_{\mathrm{e}}$.

Four motion patterns are observed for the horizontal rotation in Fig. $5 \mathrm{~b}$. For $R_{\mathrm{e}}$ below $\sim 0.07$, the egg cell oscillated around its initial position (Mode I). For $R_{\mathrm{e}}$ in the range $0.07-0.25$, it oscillated and was in contact with the cylinder because the horizontal swirl flow was not sufficiently rapid to lift it from the bottom (Mode II). For $R_{\mathrm{e}}$ above 0.25, it rotated around the horizontal axis because the swirl flow became sufficiently rapid to lift it, although the horizontal translation toward the $-X_{\mathrm{p}}$ direction occurred simultaneously (Modes III and IV). The horizontal rotation requires larger $R_{\mathrm{e}}$ than the vertical rotation does because additional energy is required for lifting it from the bottom. If excessive energy is supplied to the vertical rotation, e.g., if $a / r_{\mathrm{c}}$ is larger than $\sim 0.2$ and $R_{\mathrm{e}}$ is larger than $\sim 0.27$, it levitates, starts to roll, and then screws in the $-X_{\mathrm{P}}$ direction (Mode III-IV). Figure $5 \mathrm{c}$ shows snapshots of the contact oscillation and the horizontal rotation.

Figure $5 \mathrm{~d}$ shows the typical range of the horizontal rotation. $X_{\mathrm{c} \_l}$ and $X_{\mathrm{c} \_u}$ are the lower and upper boundaries, respectively, of $X_{\mathrm{c}} . \Delta X_{\mathrm{c}}$ is the length between the boundaries, defined by $\mid X_{\mathrm{c} u}-X_{\mathrm{c} l} l$. The egg cell does not rotate outside this range. We posited that the tapering and the bent shape of the oscillating pipette provide the lower limit of $X_{\mathrm{c}_{-} l}$, although the upper limit of $X_{\mathrm{c}_{-} u}$ is determined by the interaction of the vertical eddy and the horizontal swirl flow. 


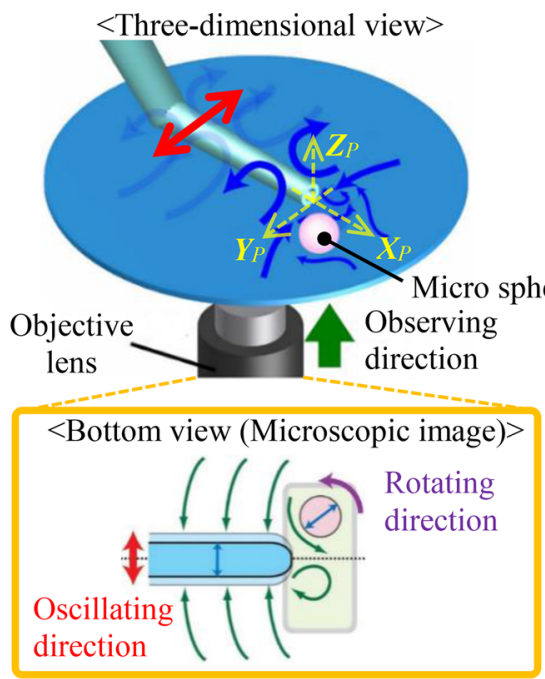

(a) Schematic of flow pattern for vertical rotation of a micro sphere.
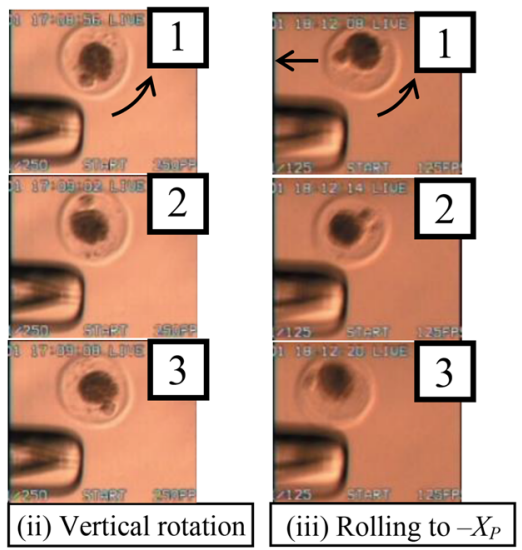

(c) Snapshots of vertical rotation.

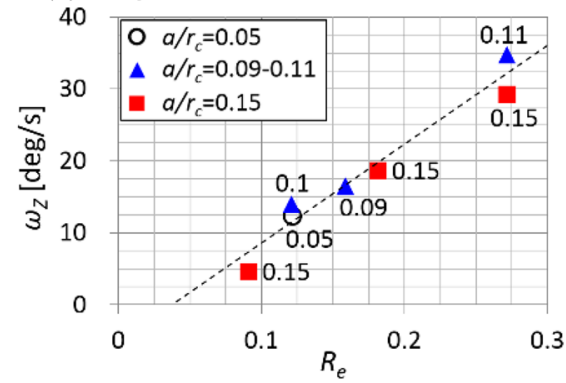

(e) Plots of $\omega_{z}, \Delta \theta_{z}$, and $L_{x y} \pm \delta r_{v}$ vs. $R_{e}$.

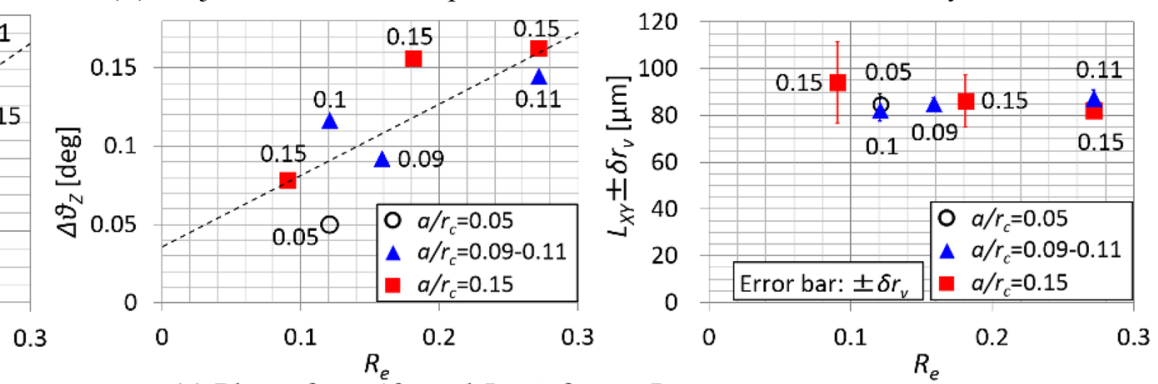

- (i) In-situ oscillation

- (ii) Vertical rotation

$\Delta$ (iii) Rolling toward -Xp direction

o Circulatory streaming

$\times$ In-situ oscillation

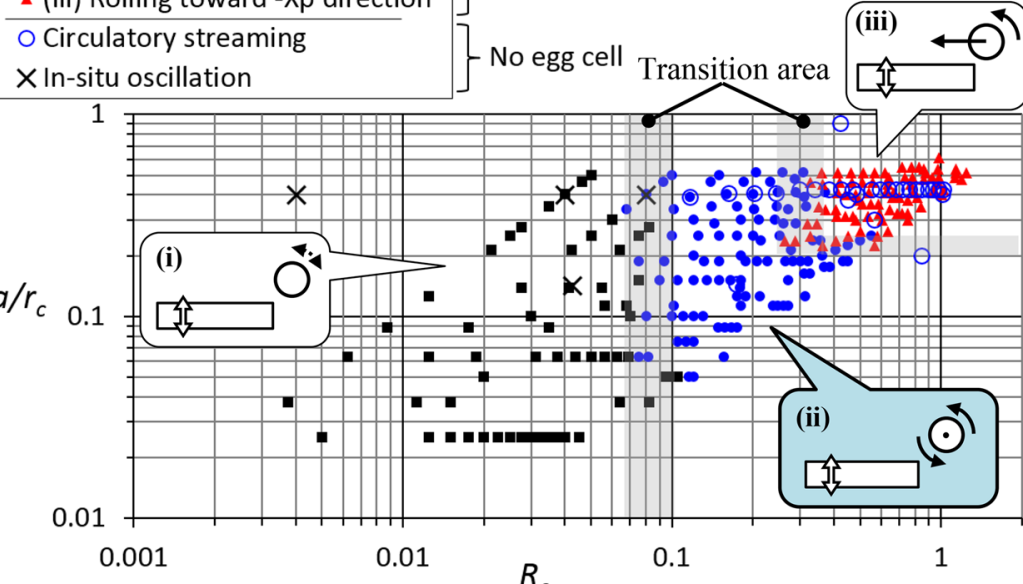

(b) Diagram of vertical rotation in the $R_{e}-a / r_{c}$ plane for $r_{e} / r_{c}=\sim 1.2$.
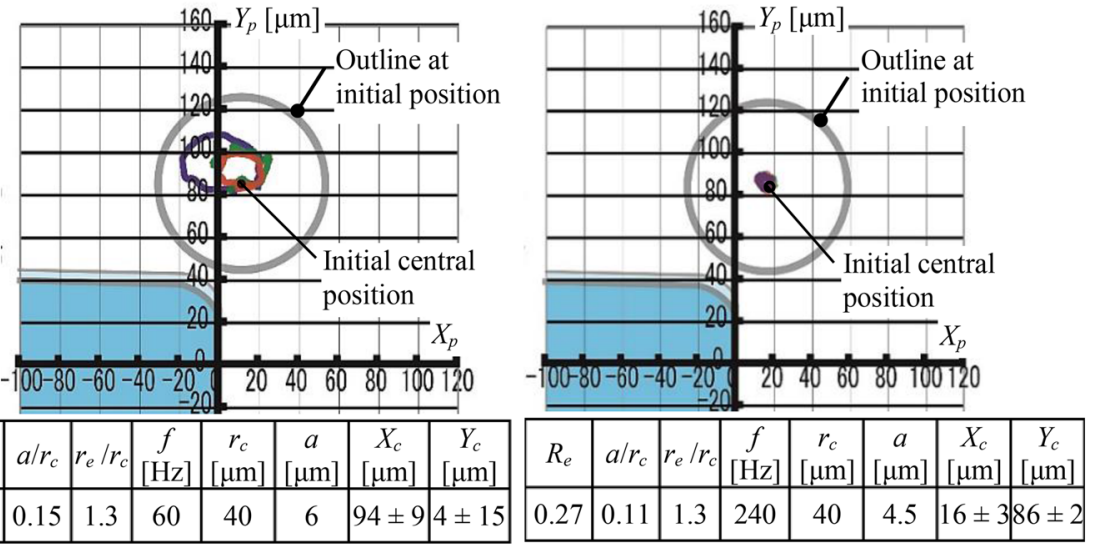

(d) Trajectories of central point with vertical rotation of $360^{\circ}, 3$ cycles.

Fig. 4 Experimental results of vertical rotations

Figure 5e shows the plots of each of $\omega_{\mathrm{h}},\left|V_{x}\right|$, and $L_{Y Z} \pm \delta r_{\mathrm{h}}$ vs. $R_{\mathrm{e}}$. Here, $\omega_{\mathrm{h}}$ is the angular velocity. $V_{x}$ is the velocity in the $X$ direction. $L_{Y Z}$ is the distance between $\left(X_{\mathrm{c}}\right.$, $0,0)$ and $\left(X_{\mathrm{c}}, Y_{\mathrm{c}}, Z_{\mathrm{c}}\right) . \delta r_{\mathrm{h}}$ is defined as the radial run out of the horizontal rotation. From the left panel of Fig. $5 \mathrm{e}, \omega_{\mathrm{h}}$ is approximately proportional to $R_{\mathrm{e}}$ as well as $\omega_{z}$. The maximum of $\omega_{\mathrm{h}}$ is $188^{\circ} / \mathrm{s}$ at $\left(R_{\mathrm{e}}, a / r_{\mathrm{c}}\right)=(1.03,0.43)$. From the middle panel of Fig. 5e, $\left|V_{x}\right|$ is approximately proportional to $R_{\mathrm{e}}$. From the right panel of Fig. 5e, $L_{Y Z}$ decreases for higher $R_{\mathrm{e}}$ as well as $L_{X Y}$, and $\delta r_{\mathrm{h}}$ was below $10 \mu \mathrm{m}$. This verifies that it does not contact the cylinder. In the supporting information, Table 4 lists the properties of the noncontact horizontal rotation. As shown in Table $4, \Delta \theta_{\mathrm{h}}$ is the rotational resolution during a cycle of the cylinder's oscillation. The minimum of $\Delta \theta_{\mathrm{h}}$ is $0.11^{\circ}$ for $\left(R_{\mathrm{e}}, a / r_{\mathrm{c}}\right)=$ $(0.28,0.18)$. 


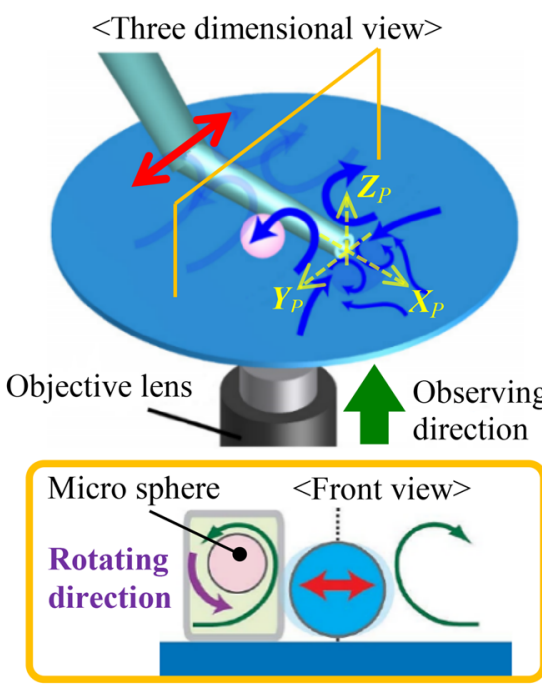

(a) Schematic of flow pattern for horizontal rotation.

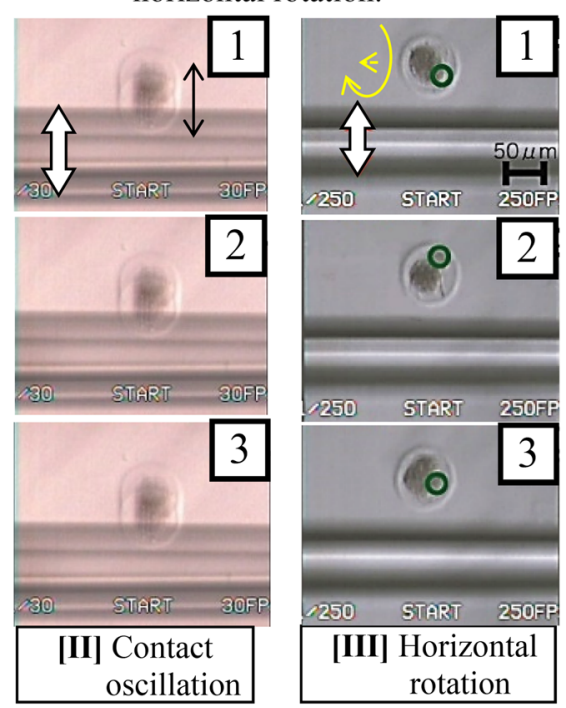

(c) Snapshots of horizontal rotation.

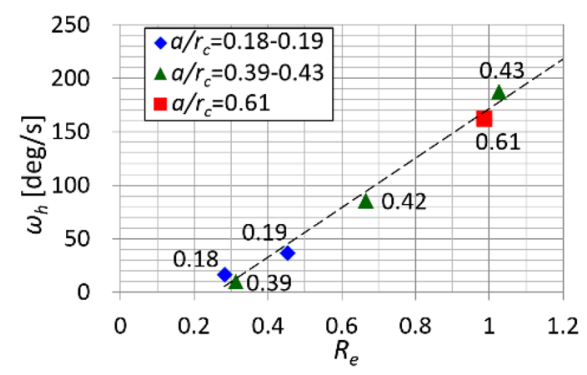

- (i) In-situ oscillation

- (ii) Vertical rotation

(iii) Rolling toward -Xp direction

$\square$ [I] In-situ oscillation, [II] Contact oscillation

$\diamond[$ III] Horizontal rotation, [IV] Horizontal rotation \& translation

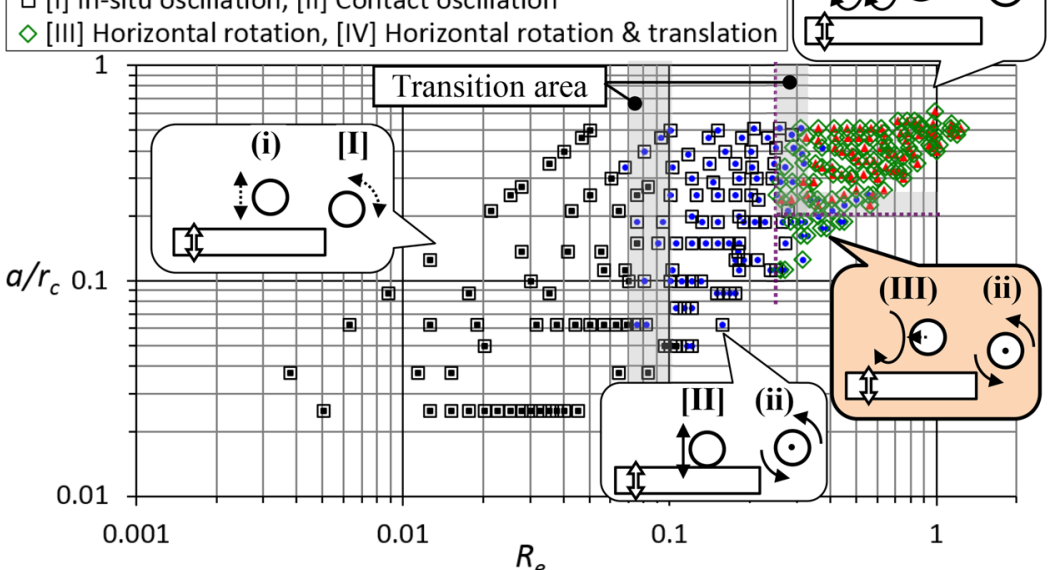

(b) Diagram of vertical and horizontal rotations in $R_{e}-a / r_{c}$ plane for $r_{e} / r_{c}=\sim 1.2$.
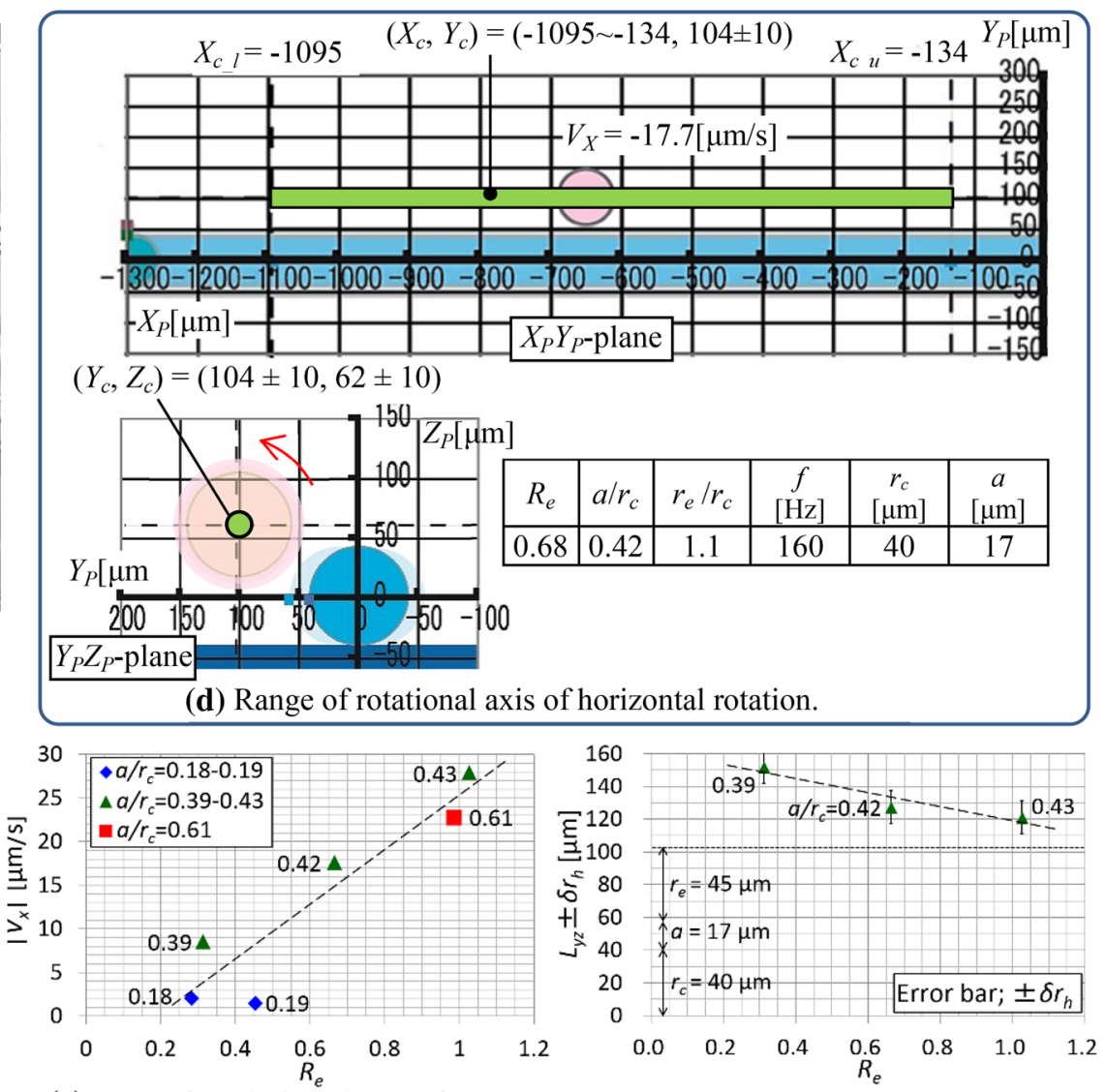

(e) Plots of $\omega_{h},\left|V_{x}\right|$, and $L_{Y Z} \pm \delta r_{h}$ vs. $R_{e}$.

Fig. 5 Experimental results of horizontal rotations

Although smaller $\Delta \theta_{\mathrm{h}}$ and higher $\omega_{\mathrm{h}}$ are important for precise and rapid microscopic operations, decreasing $\left|V_{x}\right|$ is also important because the horizontal translation is undesirable for regulating the rotational angle. We concluded that the region with $R_{\mathrm{e}}=0.25-0.5$ and $a / r_{\mathrm{c}}=$ 
0.1-0.2 (Mode III in Fig. 5b) is appropriate for the horizontal rotation.

\subsection{Drawing}

Figure 6a schematically shows the flow pattern for the drawing. Figure $6 \mathrm{~b}$ shows the typical trajectories from various initial positions along the $Y_{\mathrm{P}}$ axis for $\left(R_{\mathrm{e}}, a / r_{\mathrm{c}}\right)=(0.68$, $0.42)$. Here, we denote the initial position as $\left(X_{\mathrm{i}}, Y_{\mathrm{i}}\right)$. It shifts to the tip of the cylinder after it reaches the side when $Y_{\mathrm{i}}$ is in the range $200-350 \mu \mathrm{m}$, although it shifts in the $-X_{\mathrm{P}}$ direction when $Y_{\mathrm{i}}$ is in the range $410-480 \mu \mathrm{m}$; this is because the range of the horizontal rotation is $X_{\mathrm{P}}<-134 \mu \mathrm{m}$, as shown in Fig. 5d. Figure $6 \mathrm{c}$ shows the plots of $t_{\mathrm{d}}$ vs. $Y_{\mathrm{i}}$. Here, $t_{\mathrm{d}}$ is the drawing time from $Y_{\mathrm{i}}$ to $Y_{\mathrm{c}}$. If we estimate that $Y_{\mathrm{i}} \log$ arithmically depends on $t_{\mathrm{d}}, t_{\mathrm{d}}$ is $25 \mathrm{~s}$ when $Y_{\mathrm{i}}=$ $500 \mu \mathrm{m} . R_{\mathrm{e}}$ should be increased for widening the range and shortening $t_{\mathrm{d}}$.

\section{Multi-axial in situ micromanipulation}

\subsection{Conventional method}

Two methods can be employed to rotate the egg cell using a holding and an injection pipettes, as shown in Fig. 7a. One amounts to randomly rotating it by ejecting and suctioning the liquid inside the holding pipette, as shown in the top panel. Another method amounts to scratching it using the injection pipette with weak suctioning by the holding pipette, as shown in the bottom panel. It is generally challenging to implement either of these methods, particularly by inexperienced personnel. In this chapter, we propose a more straightforward, safer, and more precise micromanipulation based on the steady streaming.

\subsection{An oscillating cylinder}

To determine the feasibility of the proposed method, we conducted microinjection supported by the oscillation of the holding pipette. Figure $7 \mathrm{~b}$ shows the trajectories of the drawing and two-axial rotations by the steady streaming. Figure 7c shows snapshots of the microinjection process. First, we positioned it near the holding pipette by drawing. Then, we rotated it around the horizontal axis until its polar body was in the focus plane. Next, we rotated it around the vertical axis until its polar body pointed downward or upward to prevent damage to the polar body by the injection pipette. Then, we captured it by suctioning the liquid using the holding pipette and injected the culture solution of M2 into the nuclei of five frozen-thawed fertilized eggs of mice to investigate the effect of the proposed method on the cells' differentiation rate.

In the supporting information, figure S1 shows the photograph of the fertilized egg cells after injection of the culture solution assisted by the steady streaming around the oscillating holding pipette. We verified that four out of the five egg cells underwent differentiation. Because the differentiation rate of frozen-thawed oocytes was reported to be $75-95 \%$ depending on the oocytes and experimental conditions

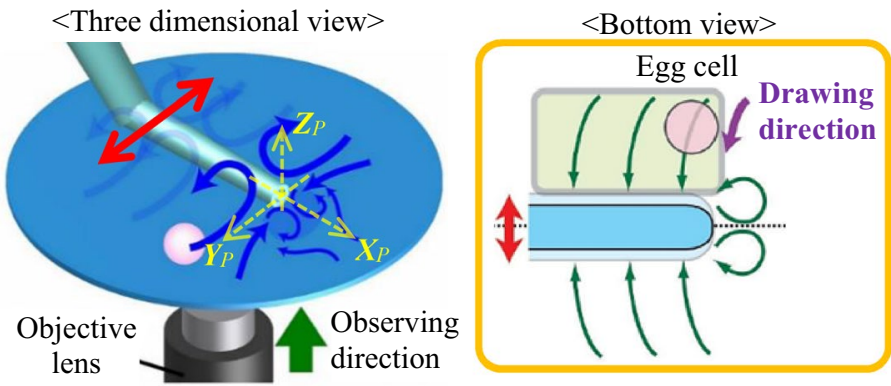

(a) Schematic of drawing of an egg cell.

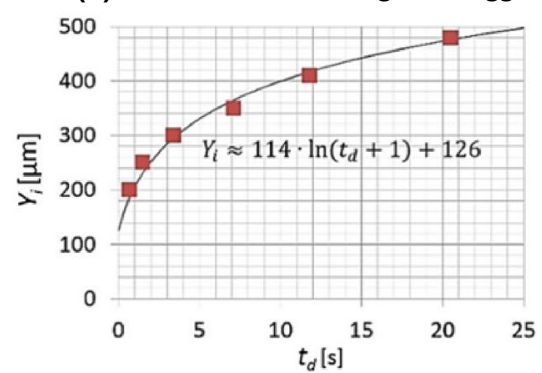

(c) Plots of $t_{d}$ vs. $Y_{i}$ with estimated logarithmic curve.

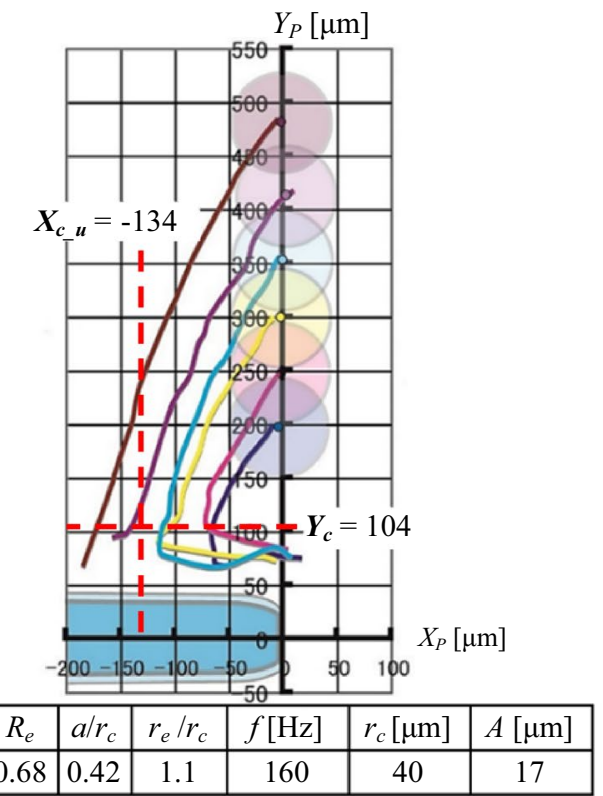

(b) Relationship between initial position and trajectory.

Fig. 6 Experimental results of drawing of an egg cell 

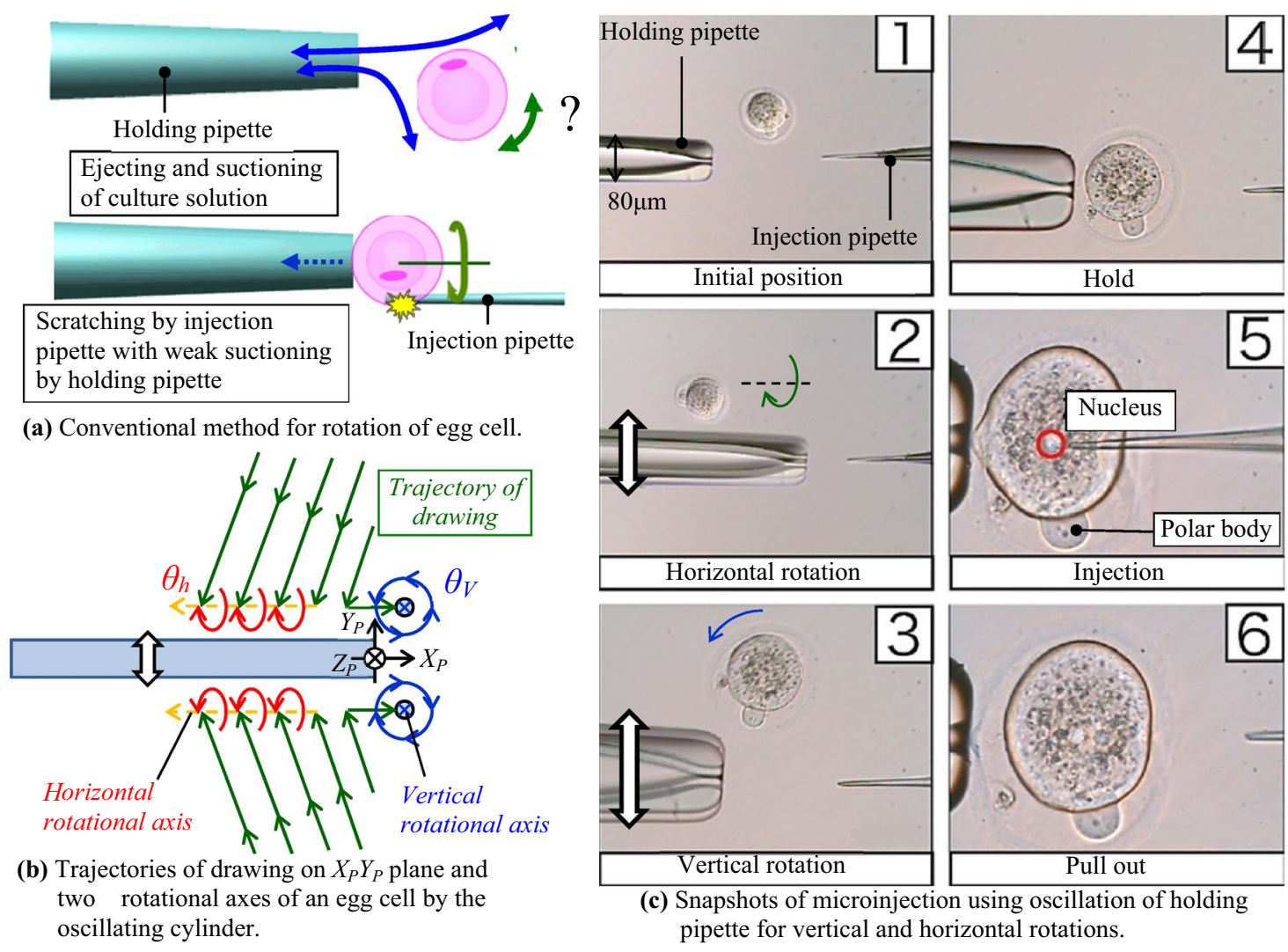

(c) Snapshots of microinjection using oscillation of holding pipette for vertical and horizontal rotations.

Fig. 7 Microinjection assisted by the steady streaming around an oscillating holding pipette

(freezing and thawing methods, culture solution, and the operator's techniques) (Nakagata 1989; Carroll et al. 1990; Borini et al. 2006; Bianchi et al. 2007), we did not find the influence of the proposed method on the differentiation rate.

The flows draw, trap, and rotate the egg cell around the vertical and horizontal axis sequentially; thus, we change $R_{\mathrm{e}}$ and $a / r_{\mathrm{c}}(f$ and $a$ ) according to the position and desired manipulation by referring to the diagram in Fig. $5 \mathrm{~b}$ and trajectories in Fig. 6b. However, during this operation, we were required to shift the holding pipette between the horizontal and rotational axes; the efficiency can be potentially increased, although additional oscillating pipettes are required.

\subsection{Two oscillating cylinders}

In this section, we describe a method for more efficient multi-axial in situ micromanipulation using two additional oscillating pipettes. Figure 8a shows an image of the setup for a microinjection. We added two holonomic miniature robots with manual $\mathrm{Z}$-axis stages for positioning the oscillating pipettes to make the manipulation compact (Fuchiwaki 2013).
Figure $8 \mathrm{~b}$ shows the trajectories of drawing toward the intersection point of the two rotational axes by combining the two oscillating pipettes. This special arrangement allows us to draw and rotate all three of the posture angles sequentially, fixing the egg cell's central point without any contact. We implemented five degrees of freedom (DoF) noncontact manipulation of the egg cell (three posture angles and two translational displacements in $X$ and $Y$ axes) with the angular resolution better than $0.05^{\circ}$ and $0.11^{\circ}$, and the radial run out of 4 and $10 \mu \mathrm{m}$ for the vertical and horizontal rotations, respectively. Because the oscillating pipettes were attached to the holonomic robots that can move $X, Y$, and $\Theta$-axes independently with positioning resolution better than $10 \mathrm{~nm}$ and $10^{-5}$ degrees, we were able to change the position and the posture of the pipettes; we were able to change the intersection point of the two rotational axes and the drawing directions, flexibly, and precisely.

Figure $8 \mathrm{c}$ shows snapshots of the microinjection process. There are two working layers. The bottom layer is for the drawing and for the horizontal and the vertical rotations by two additional oscillating pipettes. The top layer is for the microinjection using the conventional holding and injection pipettes. First, we positioned the cell near the two oscillating pipettes and the holding pipette in the bottom layer by 
Holding pipette on $X Y Z$ stage Injection pipette on $X Y Z$ stage

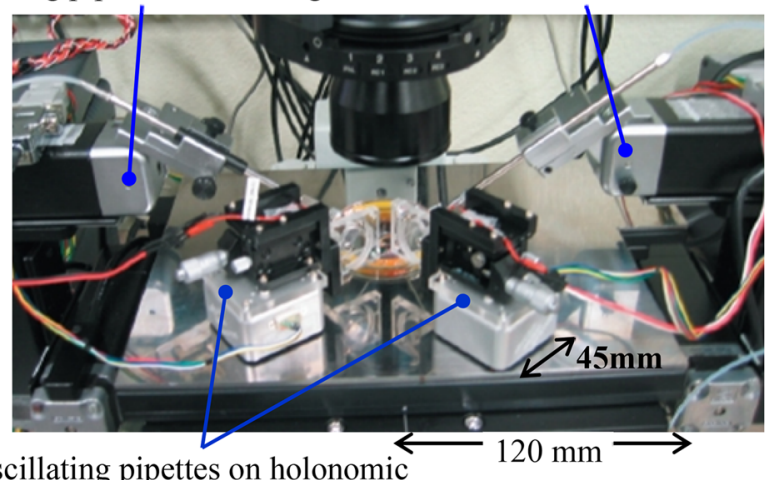

Oscillating pipettes on holonomic robots $(X Y \Theta)$ with manual $Z$ stage

(a) Image of microinjection assisted by two additional oscillating pipettes positioned by holonomic miniature robots on the inverted microscope.

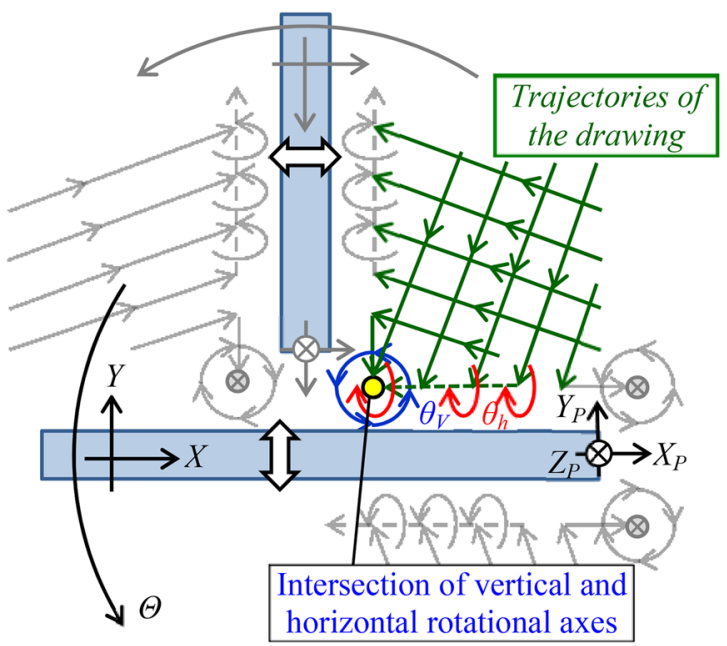

(b) Trajectories of drawing on $X_{P} Y_{P}$ plane and two axial rotations of the egg cell by two oscillating cylinders
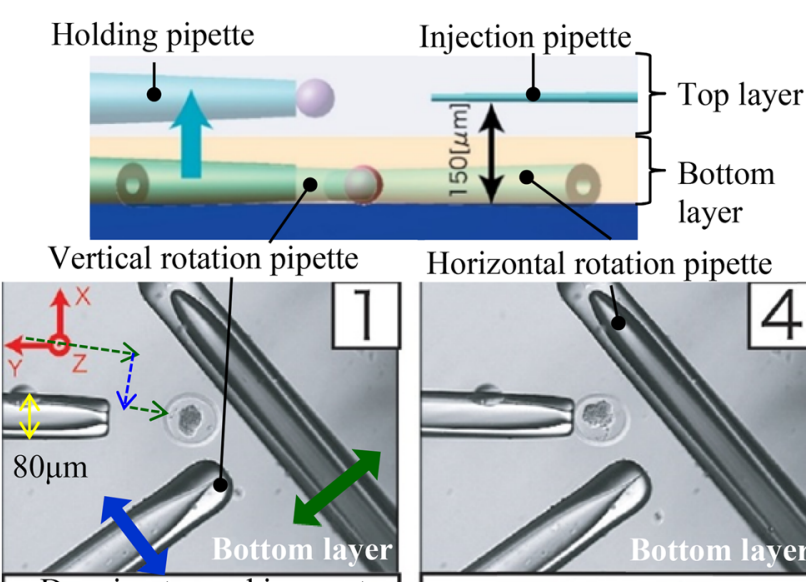

Horizontal rotation pipette
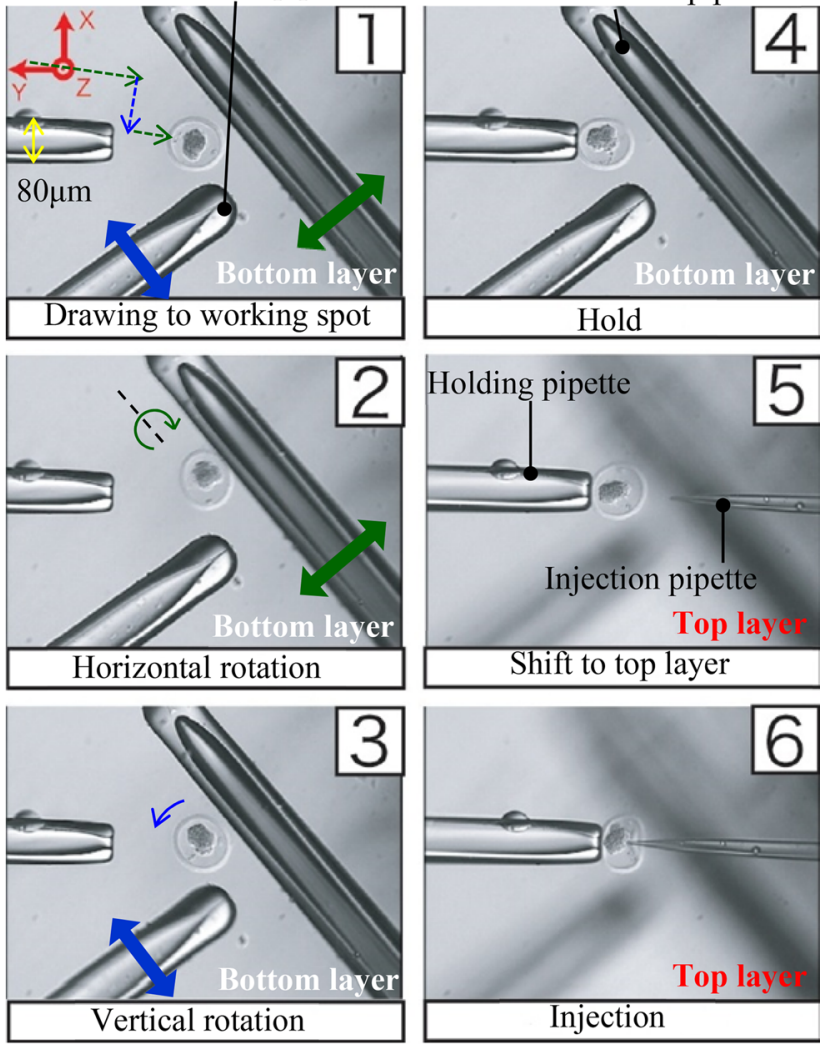

(c) Snapshots of microinjection using two additional oscillating pipettes.

Fig. 8 Multi-axial non-contact in situ micromanipulation by the steady streaming around a pair of oscillating cylinders

drawing. Next, we rotated it around the horizontal axis until its polar body was in the focus plane. Then, we rotated it around the vertical axis until its polar body pointed downward or upward. Finally, we captured it using the holding pipette and lifted the cell to the top layer for performing the injection.

The average processing time required by four inexperienced personnel was $15 \mathrm{~s}$, although the same task required 60-300 s when using the conventional methods in Fig. 7a. Thus, the proposed method can improve the processing time, particularly for inexperienced personnel.

\subsection{Discussion}

In the supporting information, Table 5 shows the specification of the multi-axial non-contact in situ micromanipulation by the steady streaming around the two oscillating cylinders on the holonomic robots for $r_{\mathrm{e}} / r_{\mathrm{c}}=\sim 1.2$. We also verified that glass and alginate spheres of diameters $\sim 100 \mu \mathrm{m}$ could be rotated around the vertical and horizontal axes and could be drawn to the pipette; the characteristics of their manipulation were similar to those of the studied egg cells. Therefore, we consider the elasticity of the targets does not give a large influence on the rotational resolution and the angular velocity, although more detailed evaluation is required. Although investigation of the operating range of the size of particles is also one of the future subjects, we estimate that sub-millimeter to the sub-micro-meter-sized particles are able to manipulated with similar characteristics under the similar conditions of $R_{\mathrm{e}}, a / r_{\mathrm{c}}$, and $r_{\mathrm{e}} / r_{\mathrm{c}}$ from the similarity law of hydrodynamics. The lower limitation should be determined by the Brownian motion. To determine the upper limitation, we should consider the influence of the gravity force, buoyancy, and inertia force on the manipulating objects 
because those volume-proportional forces are not negligible compared with the surface-proportional viscous drag force generated by the steady streaming for supra-mm-sized objects. The oscillating cylinder-based fluidic manipulation is feasible for multi-axial non-contact in situ manipulation of micro-objects with different shapes, rigidity, magnetic and optical properties.

\section{Summary and future prospects}

We described the basic principle, characteristics, and experimental results of the multi-axial micromanipulation method based on the steady streaming generated around an oscillating cylinder. In our experiments, we precisely rotated an egg cell around vertical and horizontal axes. We showed that the conditions of the rotations are determined by $R_{\mathrm{e}}$ and the ratio of a half of the amplitude to the radius of the cylinder, $a / r_{\mathrm{c}}$. The minimal angular resolutions were $0.05^{\circ}$ and $0.11^{\circ}$ and the maximal angular velocities were $34.8 \%$ s and $188^{\circ} / \mathrm{s}$ for the vertical and horizontal rotations, respectively. We also investigated the range and required time for drawing it to the cylinder. To demonstrate the feasibility, we conducted microinjection using the oscillating pipette. We were able to control its five DoF, consisting of three posture angles and two translational displacements in $X$ and $Y$ axes, without any contact.

We also explain the special arrangement of the two oscillating pipettes on holonomic miniature robots. The arrangement permitted us to draw it toward the point of intersection of the two rotational axes and also to regulate its three posture angles sequentially by fixing the central point. The average processing time of microinjection by four inexperienced personnel decreased to $15 \mathrm{~s}$ from 60 to $300 \mathrm{~s}$.

The proposed method enables a delicate, multi-axial, noncontact, in situ, and compact micromanipulation independent of the electrical, optical, magnetic, shape, and stiffness properties of the microscopic objects and which can be used in microfluidics, biomedical, and microassembly applications. Although investigation of the operating range of the size of particles is one of the future subjects, we estimate that sub-milli-meter to sub-micro-meter-sized heterogeneous particles could also be manipulated with similar characteristics under the similar conditions of $R_{\mathrm{e}}, a / r_{\mathrm{c}}$, and $r_{\mathrm{e}} / r_{\mathrm{c}}$ from the similarity law of hydrodynamics.

Future work will aim at tailoring this microscopic manipulation method to applications in microfluidics, biomedicine, and microscale assembly.

Acknowledgements We wish to thank Mr. Naoto Chiba for providing a significant amount of experimental data over 3 years. We also thank Dr. Shizue Iwasaki for the support and for providing the experimental data related to the processing of egg cells, which aided us to apply our technology to biomedical problems. We also thank Dr. Binghu Piao for computing the alternating torque and forces exerted on the cell by an oscillating cylinder, which aided us to understand the elusive microfluidics phenomena. This work was partially supported by the Industrial Technology Research Grant Program (Grant for Young Researchers) of New Energy and Industrial Technology Development Organization (NEDO), Japan (2004-2006).

\section{Compliance with ethical standards}

Conflict of interest The authors declare that they have no conflict of interest.

\section{References}

Amit R, Abadi A, Kosa G (2016) Characterization of steady streaming for a particle manipulation system. Biomed Microdevices 18:39. https://doi.org/10.1007/s10544-016-0055-1

Aoyama H, Fuchiwaki O (2001) Flexible micro-processing by multiple micro robots in SEM. Proc ICRA:3429-3434. https://doi. org/10.1109/ROBOT.2001.933148

Aoyama H, Chiba N, Fuchiwaki O et al (2006) Non-contact bio cell manipulation by nonlinear micro flow around the vibrated pipette on micro robot. In: Proc of ASPE Annual Meeting, Poster sessions mechatronics-1

Benhal P, Chase JG, Gaynor P et al (2014) AC Electric field induced dipole-based on-chip 3D cell rotation. Lab Chip 14(15):27172727. https://doi.org/10.1039/c4lc00312h

Bianchi V et al (2007) Differential sucrose concentration during dehydration $(0.2 \mathrm{~mol} / \mathrm{l})$ and rehydration $(0.3 \mathrm{~mol} / \mathrm{l})$ increases the implantation rate of frozen human oocytes. RBM Online 14(1):64-71

Borini A et al (2006) Clinical outcome of oocyte cryopreservation after slow cooling with a protocol utilizing a high sucrose concentration. Hum Reprod 21(2):512-517. https://doi. org/10.1093/humrep/dei346

Boudaoud M, Haddab Y, Gorrec YL (2012) Modeling and optimal force control of a nonlinear electrostatic microgripper. TMECH 18(3):1130-1139. https://doi.org/10.1109/TMECH.2012.21972 16

Büyükkoçak S, Özer MB, Çetin B (2014) Numerical modeling of ultrasonic particle manipulation for microfluidic applications. Microfluid Nanofluid 17(6):1025-1037. https://doi.org/10.1007/ s10404-014-1398-7

Carroll J et al (1990) Freeze-thaw-induced changes of the zona pellucida explains decreased rates of fertilization in frozen-thawed mouse oocytes. J Reprod Fertil 90:547-553

Cecil J, Kumar MBBR, Lu Y et al (2016) A review of micro-devices assembly techniques and technology. Int $\mathbf{J}$ Adv Manuf Tech 83(9):1569-1581. https://doi.org/10.1007/s00170-015-7698-6

Chen T, Sun L, Chen L et al (2010) A hybrid-type electrostatically driven microgripper with an integrated vacuum tool. Sensor Actuatators A-Phys 158(2):320-327. https://doi.org/10.1016/j. sna.2010.01.001

Chen H, Wang C, Sun D (2013) Dynamics calibration of optically trapped cells with adaptive control technology. Proc ICRA. https ://doi.org/10.1109/ICRA.2013.6630964

Chowdhury S, Thakur A, Wang C et al (2013) Automated indirect manipulation of irregular shaped cells with optical tweezers for studying collective cell migration. Proc ICRA. https://doi. org/10.1109/ICRA.2013.6630962

Coenen W (2016) Steady streaming around a cylinder pair. Proc R Soc A 472(2195):20160522. https://doi.org/10.1098/rspa.2016.0522 
Destgeera G, Sung HJ (2015) Recent advances in microfluidic actuation and micro-object manipulation via surface acoustic waves. Lab Chip 15:2722-2738. https://doi.org/10.1039/c5lc00265f

Elbuken C, Khamesee MB, Yavuz M (2009) Design and implementation of a micromanipulation system using a magnetically levitated. MEMS Robot TMECH 14:4. https://doi.org/10.1109/TMECH .2009 .2023648

Fan Z, Wang L, Rong W, Sun L (2015) Dropwise condensation on a hydrophobic probe-tip for manipulating micro-objects. Appl Phys Lett 106:084105. https://doi.org/10.1063/1.4913849

Fatikow S, Seyfried J, Fahlbusch S et al (2000) A flexible microrobotbased microassembly station. J Intell Robot Syst 27(1):135-169. https://doi.org/10.1023/A:1008106331459

Floyd S, Pawashe C, Sitti (2009) Two-dimensional contact and noncontact micromanipulation in liquid using an untethered mobile magnetic microrobot. M TRO 25(6):1332-1342. https://doi. org/10.1109/TRO.2009.2028761

Friend J, Yeo L (2011) Microscale acoustofluidics: microfluidics driven via acoustics and ultrasonics. Rev Mod Phys 83(2):647-687. https ://doi.org/10.1103/RevModPhys.83.647

Fuchiwaki O (2013) Insect-sized holonomic robots for precise, omnidirectional, and flexible microscopic processing: identification, design, development, and basic experiments. PE 37(1):88-106. https://doi.org/10.1016/j.precisioneng.2012.07.004

Fuchiwaki O, Kumagai K (2013) Development of wet tweezers based on capillary force for complex-shaped and heterogeneous microassembly. Proc IROS. https://doi.org/10.1109/IROS.2013.66964 73

Fuchiwaki O, Ito A, Misaki D, Aoyama H (2008) Multi-axial micromanipulation organized by versatile micro robots and micro tweezers. Proc ICRA. https://doi.org/10.1109/ROBOT.2008.4543318

Fuchiwaki O, Chiba N, Aoyama H (2010) U.S. Patent 2010 US 7726210

Fuchiwaki O, Arafuka K, Omura S (2012) Development of 3-DOF inchworm mechanism for flexible, compact, low-inertia, and omnidirectional precise positioning: dynamical analysis and improvement of the maximum velocity within no slip of electromagnets. TMECH 17(4):697-708. https://doi.org/10.1109/ TMECH.2011.2118764

Fuchiwaki O, Yatsurugi M, Sato T (2014) The basic performance of a miniature omnidirectional 6-legged inchworm robot from $\mathrm{cm}$ - to $\mu \mathrm{m}$-scale precise positioning. Trans Mat Res Soc Japan 39(2):211-215. https://doi.org/10.14723/tmrsj.39.211

Fuchiwaki O, Yamagiwa T, Omura S, Hara Y (2015) In-situ repetitive calibration of microscopic probes maneuvered by holonomic inchworm robot for flexible microscopic operations. Proc IROS. https://doi.org/10.1109/IROS.2015.7353561

Gijs MAM (2004) Magnetic bead handling on-chip: new opportunities for analytical applications. Microfluid Nanofluid 1(1):22-40. https ://doi.org/10.1007/s10404-004-0010-y

Grier GD (2003) A revolution in optical manipulation. Nature 424:810-816. https://doi.org/10.1038/nature01935

Hatamura Y, Nakao M, Sato T (1995) Construction of nano manufacturing world. Microsyst Technol 1:155-162

Hattori T, Kamiyama K, Kojima M et al (2015) Generation of swirl flow by needle vibration for micro manipulation. Proc IROS. https ://doi.org/10.1109/IROS.2015.7353459

Hayakawa T, Sakuma S, Fukuhara T et al (2014) A single cell extraction chip using vibration-induced

Hayakawa T et al (2015) On-chip cell transportation based on vibration-induced local flow in open chip environment. Proc IROS. https://doi.org/10.1109/IROS.2015.7353552

Hyakutake T, Nagai S (2015) Numerical simulation of red blood cell distributions in three-dimensional microvascular bifurcations. Microvasc Res 97:115-123. https://doi.org/10.1016/j. mvr.2014.10.001
Hyakutake T, Suzuki H, Yamamoto S (2015) Effect ofnon-Newtonian fluid properties on bovine sperm motility. J Biomech 48:29412947. https://doi.org/10.1016/j.jbiomech.2015.08.005

Jeong JS, Lee JW, Lee CY et al (2011) Particle manipulation in a microfluidic channel using acoustic trap. Biomed Microdevices 13(4):779-788. https://doi.org/10.1007/s10544-011-9548-0

Kim K, Liu X, Zhang Y, Sun Y (2008) Nanonewton force-controlled manipulation of biological cells using a monolithic MEMS microgripper with two-axis force feedback. J Micromech Microeng 18(5):055013. https://doi.org/10.1088/09601317/18/5/055013

Kumagai K, Fuchiwaki O (2012) A development of dispenser for highviscosity liquid and pick and place of micro objects using capillary force. KEM 516:48-53. https://doi.org/10.4028/www.scien tific.net/KEM.516.48

Leibacher I, Hahn P, Dual J (2015) Acoustophoretic cell and particle trapping on microfluidic sharp edges. Microfluid Nanofluid 19(4):923-933. https://doi.org/10.1007/s10404-015-1621-1

Leung C, Lu Z, Zhang XP et al (2012) Three-dimensional rotation of mouse embryos. IEEE Trans Biomed Eng 59(4):1049-1056 https ://doi.org/10.1109/TBME.2012.2182995

Lieu VH, House TA, Schwartz DT et al (2012) Hydrodynamic tweezers: impact of design geometry on flow and microparticle trapping. Anal Chem 84:1963-1968. https://doi.org/10.1021/ac203 $002 \mathrm{z}$

Misaki D, Naoto C, Takeshi U, Fuchiwaki O et al (2004) 3D micro image recognition for in bio-cell operation by micro robots. In: Proceedings of ASPE Annual Meeting, pp 273-276

Misaki D, Oguri A, Zhu N, Fuchiwaki O et al (2007) 3D model reconstruction with non-contact rotation mechanism using micro robots. In: Proceedings of ASPE Annual Meeting, pp 260-263

Nakagata N (1989) High survival rate of unfertilized mouse oocytes after vitrification. J Reprod Fertil 87:479-483

Nuriev A, Zaitseva O (2014) Analysis of the secondary stationary flow around an oscillating circular cylinder. Int J Mech Aero Ind Mechatron Manuf Eng 8:11. https://doi.org/10.1999/1307$6892 / 9999758$

Obata KJ, Motokado T, Saito S et al (2004) A scheme for micro-manipulation based on capillary force. J Fluid Mech 498:113-121. https ://doi.org/10.1017/S0022112003006955

Ozkan M, Wang M, Ozkan C et al (2003) Optical manipulation of objects and biological cells in microfluidic devices. Biomed Microdevices 5:61-67. https://doi.org/10.1023/A:1024467417471

Rong W, Fan Z, Wang L et al (2014) A vacuum microgripping tool with integrated vibration releasing capability. Rev Sci Instrum 85:085002. https://doi.org/10.1063/1.4891695

Sadhal SS (2012) Acoustofluidics 13: analysis of acoustic streaming by perturbation methods. Lab Chip 12:2292-2300. https://doi. org/10.1039/c2lc40202e

Savia M, Koivo HN (2009) Contact micromanipulation-survey of strategies. TMECH 14(4):504-514. https://doi.org/10.1109/ TMECH.2008.2011986

Shafiee H, Caldwell JL, Sano MB et al (2009) Contactless dielectrophoresis: a new technique for cell manipulation. Biomed Microdevices 11:997-1006. https://doi.org/10.1007/s10544-009-9317-5

Tamadazte B, Piat NL, Dembele S (2011) Robotic micromanipulation and microassembly using monoview and multiscale visual servoing. TMECH 16(2):277-287. https://doi.org/10.1109/TMECH .2010 .2040900

Tatsuno M (1973) Circulatory streaming around an oscillating circular cylinder at low reynolds numbers. J Phys Soc Jpn 35:915-920

Tatsuno M (1981) Secondary flow induced by a circular cylinder performing unharmonic oscillation. J Phys Soc Jpn 50:330-337

Walker R, Gralinski I, Lay KK et al (2012) Particle manipulation using an ultrasonic micro-gripper. Appl Phys Lett 101:163504. https:// doi.org/10.1063/1.4759127 
Wang CY, Drachman B (1982) The steady streaming generated by a vibrating plate parallel to a fixed plate. Appl Sci Res 39(1):55-68

Whirling Flow and a Thermo-Responsive Gel Pattern. Micromachines 5(3):681-696. https://doi.org/10.3390/mi5030681

Wiklund M, Green R, Ohlin M (2012) Acoustofluidics 14: applications of acoustic streaming in microfluidic devices. Lab Chip 12:24382451. https://doi.org/10.1039/c2lc40203c

Xie H, Regnier S (2011) Development of a flexible robotic system for multiscale applications of micro/nanoscale manipulation and assembly. TMECH 16(2):266-276. https://doi.org/10.1109/ TMECH.2010.2040483

Yabugaki H, Ohara K, Kojima M et al (2013) Automated stable grasping with two-fingered microhand using micro force sensor. Proc ICRA. https://doi.org/10.1109/ICRA.2013.6630959

Yalikun Y, Kanda Y, Morishima K (2016a) Hydrodynamic vertical rotation method for a single cell in an open space. Microfluid Nanofluid 20(74) https://doi.org/10.1007/s10404-016-1737-y

Yalikun Y, Kanda Y, Morishima K (2016b) A method of three-dimensional micro-rotational flow generation for biological applications. Micromachines 7(8):140. https://doi.org/10.3390/mi7080140
Ye Z, Diller E, Sitti M (2012) Micro-manipulation using rotational fluid flows induced by remote magnetic micromanipulators. J Appl Phys 112(064912). https://doi.org/10.1063/1.4754521

Yesin KB, Vollmers K, Nelson BJ (2006) Modeling and control of untethered biomicrorobots in a fluidic environment using electromagnetic fields. Robot Res 25(5-6):527-536. https://doi. org/10.1177/0278364906065389

Zhang Y, Chen BK, Liu X, Sun Y (2010) autonomous robotic pickand-place of microobjects. TRO 26(1):200-207. https://doi. org/10.1109/TRO.2009.2034831

Publisher's Note Springer Nature remains neutral with regard to jurisdictional claims in published maps and institutional affiliations. 